\title{
Modeling Truncated Hemoglobin vibrational dynamics
}

\author{
Luca Marsella* \\ International School for Advanced Studies (S.I.S.S.A.), \\ via Beirut 2-4 34014 Trieste, Italy
}

(Dated: February 8, 2020)

\begin{abstract}
We present a study on the near equilibrium dynamics of two small proteins in the family of truncated hemoglobins, developed under the framework of a Gaussian network approach. Effective beta carbon atoms are taken into account besides $C^{\alpha} \mathrm{S}$ for all residues but glycines in the coarse-graining procedure, without leading to an increase in the degrees of freedom ( $\beta$ Gaussian Model). Normalized covariance matrix and deformation along slowest modes with collective character are analyzed, pointing out anti-correlations between functionally relevant sites for the proteins under study. In particular we underline the functional motions of an extended tunnel-cavity system running inside the protein matrix, which provide a pathway for small ligands binding with the iron in the heme group. We give a rough estimate of the order of magnitude of the relaxation times of the slowest two overdamped modes and compare results with previous studies on globins.
\end{abstract}

Keywords: Truncated Hemoglobins; oxygen transport; Gaussian models; vibrational properties of proteins; overdamped dynamics.

\footnotetext{
*E-mail: marsella@sissa.it
} 


\section{INTRODUCTION}

Several studies in the past decades have shown the validity of the normal modes approach to extract useful information on the large-scale functional movements of proteins near their native state conformation [1, 2, 3, 4, 15].

Molecular dynamics simulations of biomolecules performed using detailed all atoms potentials yield lots of information regarding large amplitude, concerted displacements of atoms [6]. However these can be simply obtained within the harmonic approximation and from the analysis of the hessian matrix: in fact only low-frequency modes provide the major part of the norm for those global motions, whereas the fastest modes account for only spatially localized fluctuations [7, 8].

It has become customary to project the dynamical trajectories of the atoms in the molecules onto normal mode axes 9]; thus one is brought to interpret the functional, large amplitude motions of biological relevance for proteins as superpositions of independent harmonic modes of oscillations of a network of atoms.

A pioneering work developed by Tirion [10] paved the way for extremely simplified Normal Mode Analysis (NMA): detailed harmonic potentials are replaced by a single-parameter, spring-like potential between atoms found to be in contact in the native configuration.

Despite the extreme simplicity of this approach, the good agreement obtained with atomic mean square displacements of molecular dynamics simulations 10| opened the possibility for further studies, within the same approach [11, 12, 13, 14, 15, 16, 17, 18]: a good level of consistency with more accurate analyses is achieved even treating proteins under coarsegrained schemes, as recently shown by Bahar and co-workers 11, 12, 13, 14, 15, 16], who developed simple yet useful models to explore the collective motions of proteins, profitably adopted by other groups [19, 20].

In the present study two structures recently solved [21] are addressed, which belong to the family of truncated hemoglobins (trHbs), small heme proteins widely distributed in bacteria, protozoa and plants, forming a distinct group within the hemoglobin superfamily [22, 23, 24].

Though having a simpler structure than the traditional globin fold, they still preserve the respiratory function, providing transport and storage of oxygen molecules. Furthermore they have been proposed to be involved also in other biological functions, such as protection 
against reactive nitrogen species, photosynthesis or to act as terminal oxidases 222, 25, 26, 27].

The low complexity of trHbs structure, compared to normal globin folds, might help the comprehension of the mechanisms used by these shorter molecules to bind small ligands to the heme iron atom (e.g.: $\mathrm{O}_{2}$, their main target, and $C O$, to which heme has a high affinity).

In particular, the presence of an apolar cavity system extending throughout the protein matrix of truncated hemoglobin from Mycobacterium Tuberculosis and homologous structures has been recently noticed [24, 28]: this tunnel connects the heme distal pocket to the protein surface, and may thus allow an efficient diffusion path for oxygen and other small molecules to the iron atom (fig 1).

The role of protein cavities has been deeply investigated in myoglobin (see [24, 29, 30] and references therein), both theoretically using computer simulations and experimentally suggesting pathways for ligands migration switched by a small number of substates, which can be allosterically converted to the stable conformations [31].

These issues are investigated here from a novel point of view, through a simple coarsegrained scheme in the spirit of the Gaussian chain models, with a twofold goal: understanding the mechanical processes involved in the functional movements of these key proteins and taking advantage of this new Gaussian framework, computationally fast and conceptually simple.

\section{STRUCTURAL CHARACTERIZATION}

The structures addressed in the present study are two truncated hemoglobins from the ciliated protozoan Paramecium Caudatum (PtrHb, pdb id: 1dlw) and the green unicellular

alga Chlamydomonas Eugametos (CtrHb, pdb id: 1dly), solved at 1.54 and $1.80 \AA$ resolution respectively, by Pesce et al. [21].

Similarly to the other proteins belonging to the trHb family, they display low sequence identity with hemoglobins from vertebrate and non vertebrate. This is smaller than $15 \%$ for $\mathrm{PtrHb}$ and $\mathrm{CtrHb}$, due to substantial residue deletions at either $\mathrm{N}$ - or $\mathrm{C}$ - termini and in the $\mathrm{C}$ and D helical region of the globin fold [21].

More than $70 \%$ of the residues in the two structures belongs to helices, mainly of type $\alpha$ (above $67 \%$ in both proteins: only the short helix $\mathrm{C}$ is of type $3_{10}$ ): this is a typical 

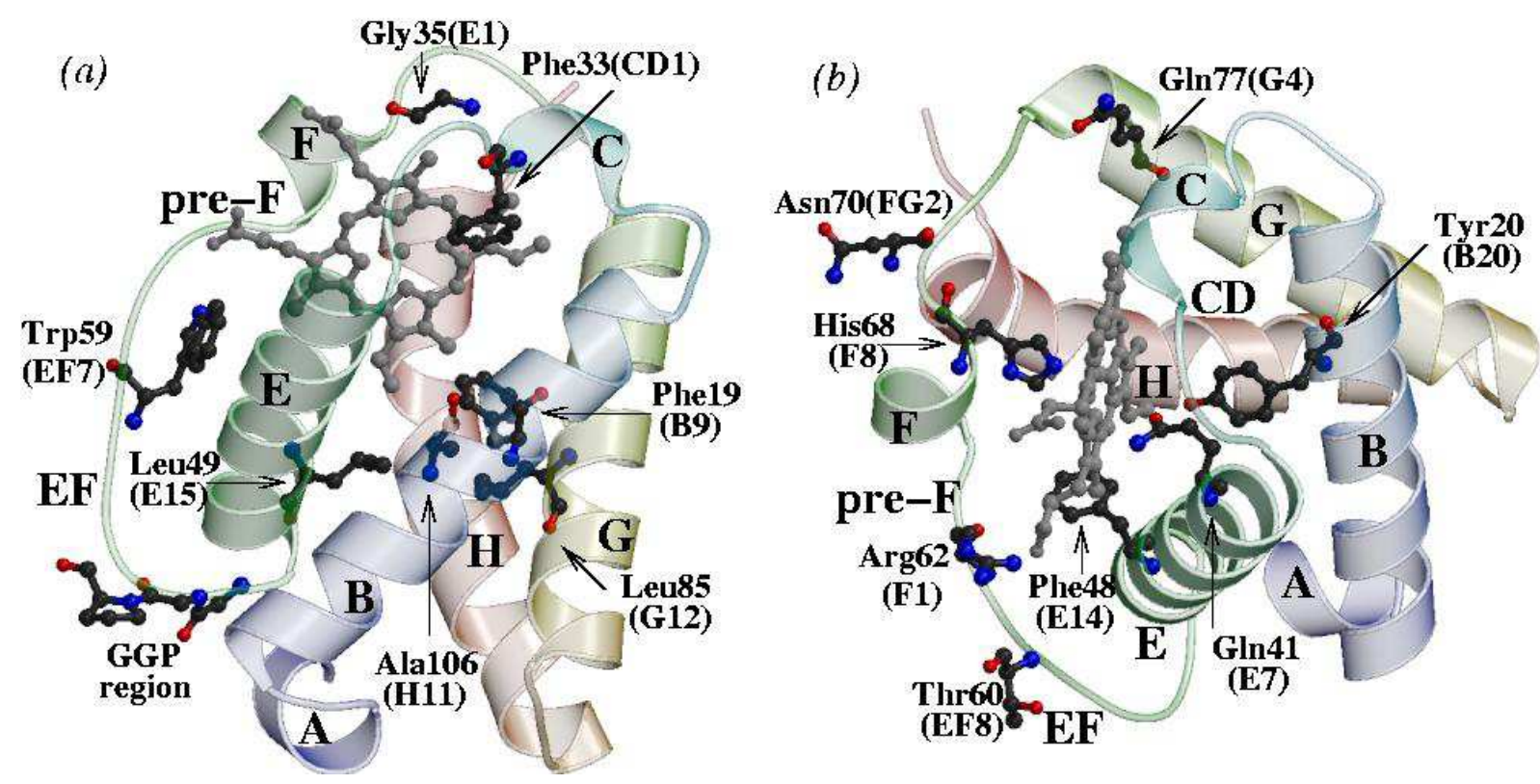

FIG. 1: Truncated hemoglobin fold from Paramecium Caudatum: helices, coils and the main residues described in the text are labeled according to the standard nomenclature for globins. The two-over-two helical structure enclosing the heme group is clearly visible: (a) side view, (b) top view. Figure drawn using Molscript [32] and Raster3d [33].

feature of the globin fold, which leads to guess a primary role of helices in the functional motions of these proteins, as well as in myoglobin and hemoglobin. Nonetheless several structural differences make truncated hemoglobins fall in a distinct group in the hemoglobin superfamily [21, 24].

Helices in the globin fold are traditionally indexed through capital letters A, B, C, D, E, F, G and H, while loops between them are named according to the nearby helices, and residues are numbered sequentially with each unit [34].

The structures taken in consideration here reveal the so called "two over two $\alpha$ helical sandwich" (fig. 11), in place of the classical "three over three" observed in the globin fold [35]: in fact helix D is absent, while N-terminal A helix and proximal $\mathrm{F}$ helix are drastically reduced to only one turn.

A structure-based sequence alignment of $\mathrm{PtrHb}, \mathrm{CtrHb}$ and other truncated hemoglobins with sperm whale Myoglobin, reported in [21], shows the strongly conserved residues among proteins in the trHbs family, mainly of three types: 
glycine rich motifs especially at helices termini, which enhance structural flexibility (GlyGly motifs at the beginning of the AB and EF regions, and Gly-Arg/Lys in the pre-F region [21]);

hydrophobic residues on heme distal and proximal sides, which play the main role of shielding heme from solvent molecules, in order to prevent iron oxidation ;

heme binding residues stabilizing the porphyrin ring in the heme pocket; one particularly relevant is the proximal histidine, His 68 , localized on helix F.

Strongly conserved residues on the distal side responsible for the shielding of the heme pocket from the solvent are mainly localized on helices B and E, as well as in the CD and EF loops: hydrophobic residues Phe A12, B9, CD1, E14 and Trp EF7, with their side chains pointing to the inner part of the molecule; Tyr B10, Gln E7, with side chains responsible for the stabilization of the ligand bound to heme [21, 24].

The hydrophobic residues identified in [21, 28] as the ones defining a cavity inside the molecule, linking the solvent exposed surface of the proteins to the heme group are positioned on the distal side. They are mainly localized on helices A (at the opening of the tunnel on the surface), B, E (limiting the distal side) and G.

On the proximal side of the heme pocket one finds the proximal histidine, in a strongly conserved position within hemoglobin $(\mathrm{Hb})$ and trHb families: the imidazole ring of histidine allows it to act as either a proton donor or acceptor at physiological pH. In hemoglobins is essential its ability to buffer the $H^{+}$ions from carbonic acid ionization in red blood cells, allowing the molecule to exchange $\mathrm{O}_{2}$ and $\mathrm{CO}_{2}$ respectively at the tissues and at the lungs [36].

It will be shown how the small $\alpha$ helix F, which contains the proximal histidine F8, can play a leading role as a reference position for elucidating the functional motions of the protein regions around the heme pocket.

\section{THEORY}

The model adopted in this study is the Beta Gaussian Model ( $\beta \mathrm{GM}$ ) presented in [19], a single parameter model apt to describe small amplitude fluctuations of residues around their native-state equilibrium: the model is based on the Gaussian Network Model (GNM) 
and on the Anisotropic Gaussian Model (ANM), which have been successfully exploited in previous studies on the functional motions of proteins [11, 12, 13, 14, 15, 16], under the framework of the single parameter model introduced by Tirion [10].

Only alpha and beta carbon atoms $\left(C^{\alpha}, C^{\beta}\right)$ are treated: rather than the actual $C^{\beta}$, the latter is an effective centroid accounting for the directionality of the side chain, built for all residues but glycines and terminus ones; its position is determined by the coordinates of neighbouring $\alpha$ carbons [19, 37], according to the following relation:

$$
\mathbf{r}_{i}^{\beta}=\mathbf{r}_{i}^{\alpha}+3 \frac{2 \mathbf{r}_{i}^{\alpha}-\mathbf{r}_{i+1}^{\alpha}-\mathbf{r}_{i-1}^{\alpha}}{\left|2 \mathbf{r}_{i}^{\alpha}-\mathbf{r}_{i+1}^{\alpha}-\mathbf{r}_{i-1}^{\alpha}\right|}
$$

where the vectors $\mathbf{r}_{i}^{\alpha}$ and $\mathbf{r}_{i}^{\beta}$ hold the native coordinates (in Angstroms) of the $\alpha$ carbon atom and of the effective $\beta$ centroid which belong to the $i$ th residue. Expanding the displacement of the $C^{\beta}$ from the equilibrium to leading order in the displacements of the $C^{\alpha} \mathrm{S}$ one gets:

$$
\delta \mathbf{r}_{i}^{\beta} \sim 3 \frac{2 \delta \mathbf{r}_{i}^{\alpha}-\delta \mathbf{r}_{i+1}^{\alpha}-\delta \mathbf{r}_{i-1}^{\alpha}}{\left|2 \mathbf{r}_{i}^{\alpha}-\mathbf{r}_{i+1}^{\alpha}-\mathbf{r}_{i-1}^{\alpha}\right|}
$$

The hamiltonian of the system depends quadratically on the deviations of the $C^{\alpha}$ and $C^{\beta}$ from their native positions, assumed to be the energy minimum in the configuration space (thus neglecting crystal effects on X-ray structures): the displacements of protein's atoms from the equilibrium position are supposed to be small enough to justify this approximation [1, 9, 14].

The hamiltonian includes interactions between $\alpha$ and $\beta$ carbons lying within a cut-off distance $r_{c}$, above which no pairwise interaction is allowed, as well as an effective interaction accounting for the strength of the peptide bond for nearest-neighbouring $C^{\alpha} \mathrm{s}$ :

$$
\mathcal{H}=\mathcal{H}^{\text {peptide }}+\mathcal{H}^{\alpha \alpha}+\mathcal{H}^{\alpha \beta}+\mathcal{H}^{\beta \beta}
$$

where

$$
\begin{aligned}
\mathcal{H}^{\text {peptide }} & =\frac{\gamma_{p}}{2} \sum_{i} \sum_{\mu, \nu} \mathcal{M}_{i i+1}^{\mu \nu}(\alpha, \alpha) \delta r_{i, \mu}^{\alpha} \delta r_{i+1, \nu}^{\alpha} \\
\mathcal{H}^{x y} & =\frac{\gamma_{x y}}{2}\left(1-\frac{\delta_{x y}}{2}\right) \sum_{i, j} \sum_{\mu, \nu} \mathcal{M}_{i j}^{\mu \nu}(x, y) \delta r_{i, \mu}^{x} \delta r_{j, \nu}^{y}
\end{aligned}
$$

$\gamma_{p}$ is the elastic constant accounting for the relative strength of the effective peptidic interaction between nearest-neighbouring $\alpha$ carbons; 
$\gamma_{x y}$ is the elastic constant for the contact interaction between carbon atoms of type $x$ and

$$
y(x, y \in\{\alpha, \beta\})
$$

$\delta_{x y}$ is Kronecker delta to avoid double counting of the interactions between atoms of the same type;

$\delta \mathbf{r}_{i}^{x}$ is the displacement from the native position of the carbon atom of type $x$ that belongs to the $i$ th residue ( $\mu$ and $\nu$ are the indexes of the Cartesian components);

$\mathcal{M}_{i j}(x, y)(i \neq j)$ is a $(3 \times 3)$ matrix, the off-diagonal super-element of the hessian matrix for the interaction between atoms of type $x$ and $y$ which belong to residues $i$ and $j$ :

$$
\mathcal{M}_{i j}^{\mu \nu}(x, y)=\Gamma_{i j}^{x y} \frac{r_{i j, \mu}^{x y} r_{i j, \nu}^{x y}}{\mathbf{r}_{i j}^{x y} \cdot \mathbf{r}_{i j}^{x y}}
$$

where $\Gamma_{i j}^{x y}(i \neq j)$ is equal to 1 if the native separation of the corresponding atoms lies below the cut-off radius $r_{c}, 0$ otherwise; $\mathbf{r}_{i j}^{x y}=\mathbf{r}_{i}^{x}-\mathbf{r}_{j}^{y}$ is the vector of native separation of atoms of type $x$ and $y$ that belong to residues $i$ and $j$ respectively. Entries of diagonal super-elements are built according to the relation:

$$
\mathcal{M}_{i i}^{\mu \nu}(x, y)=-\sum_{j \neq i} M_{i j}^{\mu \nu}(x, y)
$$

Since the position of the effective $C^{\beta}$ and its displacement from equilibrium are fully determined by $\alpha$ carbons coordinates ( equations (11) and (2) ), by substitution of (10) and (2) in (4) one is left with an effective hamiltonian $\tilde{\mathcal{H}}$ which depends quadratically on $C^{\alpha}$ displacements from native state [19] (the index of atom type will be therefore dropped in the following equations for simplicity):

$$
\tilde{\mathcal{H}}=\frac{\gamma}{2} \sum_{i j} \sum_{\mu \nu} \tilde{\mathcal{M}}_{i j}^{\mu \nu} \delta r_{i, \mu} \delta r_{j, \nu}
$$

where $\gamma_{p}$ and $\gamma_{x y}(x, y \in\{\alpha, \beta\})$ have been incorporated in $\tilde{\mathcal{M}}_{i j}$, expressed in units of the reference elastic constant $\gamma$.

Time dependent two-point correlation functions can be calculated within a Langevin dynamics leading to equilibrium with the Boltzmann factor $e^{-\beta \tilde{\mathcal{H}}}[12]$. In the overdamped regime with the viscous damping factor $f$, the same for all residues [11], and white noise $\eta_{i}(t)$, the Langevin equation for our system is [38]:

$$
f \frac{d}{d t} \delta r_{i, \mu}(t)+\gamma \sum_{j, \nu} \tilde{\mathcal{M}}_{i j}^{\mu \nu} \delta r_{j, \nu}(t)=\eta_{i}(t)
$$


One can easily get from equation (8) the time dependence of cross correlations between couples of $C^{\alpha}$ s (the so-called "reduced" cross-correlations):

$$
\left\langle\delta \mathbf{r}_{i}(t) \cdot \delta \mathbf{r}_{j}(0)\right\rangle=\frac{k_{B} T}{\gamma} \sum_{k} \frac{1}{\lambda_{k}}\left(\mathbf{a}_{i k} \cdot \mathbf{a}_{j k}\right) e^{-\lambda_{k} \frac{t}{\tau}}
$$

$\tau=\frac{f}{\gamma}$ is the reference relaxation time, corresponding to an overdamped spring of elastic constant $\gamma$ in a dissipative medium of friction $f ; \lambda_{k}$ are non zero eigenvalues of $\tilde{\mathcal{M}}$ and $\mathbf{a}_{k}$ the corresponding eigenvectors.

Theoretical B-factors (measured in $\AA^{2}$ ) are obtained from the diagonal elements of the reduced covariance matrix (i.e. from the mean square fluctuations of $C^{\alpha}$ s around native-state equilibrium, after thermal equilibrium has been reached), through the relation:

$$
B_{i}=\frac{8 \pi^{2}}{3} \frac{k T}{\gamma}\left\langle\delta \mathbf{r}_{i} \cdot \delta \mathbf{r}_{i}\right\rangle
$$

Equation (10) will be used to fit the experimental B-factors and get an estimate of the elastic constant $\gamma$.

\section{A. Tuning model parameters}

In order to obtain reliable data for the structures under study, we compare theoretical and experimental results using the ranking correlation between the two data sets as a guideline to tune model parameters to their optimal values.

ANM was applied on the structures as well as $\beta \mathrm{GM}$ : in the case of the trHb, the theoretical temperature factors obtained with the $\beta \mathrm{GM}$ showed a higher value of Kendall's non parametric $\tau$ [39] (see below) against the experimental ones ( $\tau=0.45$ for ANM with $r_{c}=13.0 \AA, \tau=0.57$ for $\beta \mathrm{GM}$ with $r_{c}=7.0 \AA$, in the case of $\left.1 \mathrm{DLW}\right)$.

ANM works very well for bigger complexes, while for smaller proteins more details are required: the reason for the better agreement obtained by the $\beta \mathrm{GM}$ is to be found in the presence of the $\beta$ centroids, which considerably increases the number of pairwise interactions and takes into account the directionality of the side chains is in the contact map of $\alpha$ carbons.

As a consequence, the $\beta \mathrm{GM}$ needs a lower and more realistic cut-off radius $r_{c}$ to reproduce experimental B-factors and molecular dynamics data, in comparison to those used by ANM [15], as already remarked in a previous study [19], even with small proteins like trHbs: hence the choice to use the $\beta \mathrm{GM}$ in the present work. 
Here in particular, the best agreement between theory and experiment was found using a cut-off of $7.0 \AA$. This choice is imposed by the difference in compactness between helical regions and coils, and it is critical in order to keep the density of effective contact interactions at the coarse-grained level comparable to the all atoms one.

Larger cut-offs cause contact density to be overestimated for the helical regions, leading to smaller values of B-factors, with respect to the experiment: the consequence is a marked difference between flexible and solvent exposed parts of the protein, compared to the less flexible and buried parts (fig. 2).

A key point was the tuning of $\gamma_{p}$, the ratio between the effective peptide bond and the $\alpha \alpha$ interaction: it accounts for the relative stiffness of the covalent bonds along the backbone as opposed to the weaker contact interactions between $C^{\alpha}$ pairs.

Summarizing the values for the parameters used in the calculation for both structures, $r_{c}=7.0 \AA, \gamma_{p}=2.0, \gamma_{\alpha \alpha}=\gamma_{\alpha \beta}=\gamma_{\beta \beta}=1.0$ (the last ones are in units of $\gamma$ ). The value of the elastic constant $\gamma$ will be determined later, fitting the results of the model with the available experimental data.

\section{B. Temperature factors and heme modeling}

Truncated hemoglobins are heme proteins, the heme group being the active site of the molecule: there oxygen and carbon oxide bind to the sixth coordination position of the iron atom, which lies at the center of the tetrapyrrole ring and is bound to the imidazole ring of the proximal histidine F8 at the fifth coordination site (His 68, eighth residue of helix F in sperm whale myoglobin and in vertebrate hemoglobins, where nomenclature "F8" comes from [40]).

Figure 2 shows the plot of the $\alpha$ carbon atoms B-factors of the X-ray structure of truncated hemoglobin in Paramecium Caudatum and their corresponding mean square displacements derived from the $\beta \mathrm{GM}$ : most mobile regions are loops and turns between helices, which on the contrary display smaller fluctuations, in agreement with the results of an NMA study performed on deoxymyoglobin $(\mathrm{Mb})$ [41, 42].

The significance of the correlation between experimental and theoretical values is deduced from Kendall's non parametric $\tau$ [39]. Since one does not know a priori the probability distribution of the experimental B-factors, a significance for the agreement between the two 


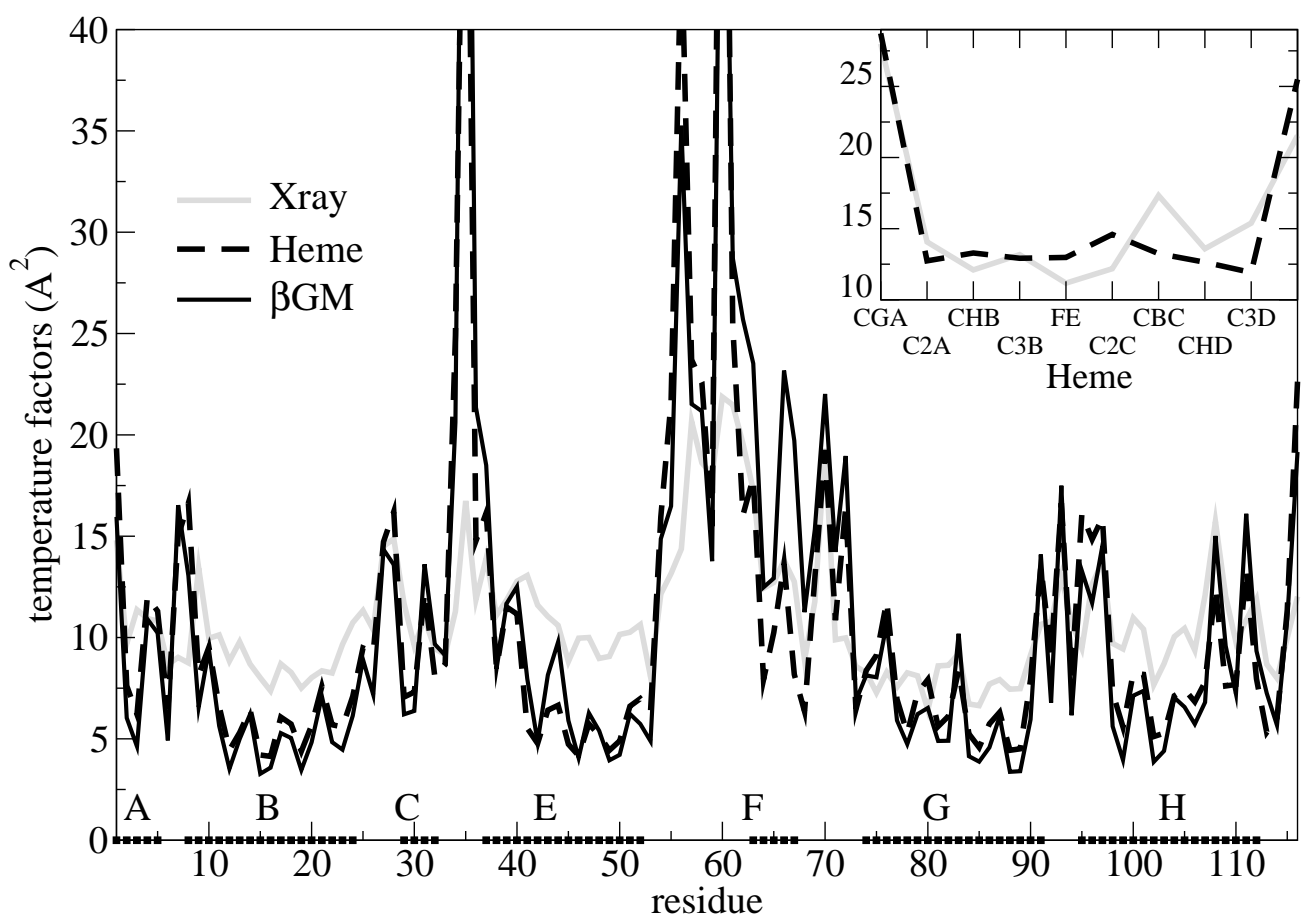

FIG. 2: Theoretical (black) versus experimental (gray) X-ray B-factors for $\alpha$ carbons in PtrHb, related through equation 10] Theoretical B-factors including coarse-grained heme group are shown for comparison (dashed). Helical segments have been marked on residues axis. The inset shows theoretical versus experimental B-factors for coarse-grained heme, with pdb names of iron and carbons included in the coarse-graining.

data sets cannot be computed from the value of the linear correlation coefficient. On the other hand, the rank correlation given by $\tau$ is independent from the distribution. Kendall's $\tau$ for $P \operatorname{trHb}$ is 0.56 (0.52 with heme), for $C \operatorname{trHb}$ is 0.37 (0.40 with heme), and $P_{\text {null }}(\tau)<10^{-9}$ in all cases $\left(P_{\text {null }}(\tau)\right.$ is the probability for two random sets of data to have a value of $\tau$ bigger than the one found between B-factors predicted by the model and calculated from X-ray structure. The number of residues is 116 for PtrHb and 121 for $\mathrm{CtrHb}$ ).

The coarse-graining on the heme group includes the iron atom and nine carbons of the porphyrin ring (whose names are reported on the $\mathrm{x}$ axis of the inset in fig. 20), chosen in order to keep the number of contacts in the modeled system comparable to the number of heme native contacts with nearby residues, thus avoiding to have a loosely connected group as an artifact of the coarse-graining procedure.

Insertion of heme brings only one relevant change to the temperature factors plot (fig. (2): 
helix F has displacements from equilibrium considerably damped, as it was expected, being bound to the iron atom. A reduction in the fluctuations is shown also by the loops between helices $\mathrm{C}$ and F, to a lesser extent than in helix F. The protein part of the reduced covariance matrix obtained including the coarse-grained heme was compared with the covariance matrix computed without modeling the tetrapyrrole ring. The two show a Kendall's parametric correlation $\tau \sim 0.81$ over more than thirteen thousands of points, which stands for a remarkable agreement between them: the coarse-grained heme in fact anticorrelates with the same parts of the protein as helix F, even if more weakly (data not shown). This is not surprising, since the iron atom and the proximal histidine F8 are in direct contact, so the motion of the heme group will be strongly correlated with the one of the F helix, following the proximal side in its deviations from native-state equilibrium: the inclusion of few more atoms under the coarse-grained scheme adopted here do not seem to significantly modify the correlations. The mechanical response of the protein upon binding of ligands on the iron atom is given by the properties of the network of backbone atoms: thus a good agreement with known behavior of globins may be achieved using gaussian models even without considering heme groups in the coarse-graining procedure [43].

The $\beta \mathrm{GM}$ heme B-factors plot is in substantial agreement with the experimental B-factors for heme (fig. 2, smaller plot). In fact the heme pocket is entirely surrounded by non polar residues: one of the main purposes of the distal region is to screen the heme group from solvent interaction, in order to avoid iron oxidation [40].

The results for Kendall's ranking lie in the typical range of gaussian models [19], even with terminal residues included, and the confidence of the correlation is no doubt statistically significant: still there are some regions of the protein whose fluctuations are not well reproduced by the model, as shown in the plot of temperature factors (fig. 21).

The model overestimates interactions between $\alpha$ and $\beta$ carbons belonging to secondary structures, resulting in local deviations from the density of the all atom picture. Hence displacements of residues belonging to helical regions are underestimated, since these are the most compact parts of the protein and it produces deviations in the profile of B-factors, whose values depend both on the assembly of secondary motifs [44] and on the local packing density [45].

Furthermore, electrostatics and solvent exposure for different residues are not taken into account by the simple approach of the model: electrostatic interactions localized on helices 
may modify the magnitude of the driving forces producing larger displacements from native state than expected.

POPS program (Parameter OPtimized Surface [46]) has been used to calculate the solvent accessible surface area per residue for PtrHb: the most exposed residues are the ones displaying the greatest average displacements from the native structure, as it was expected (figg. 2, 5). These small residues (Gly 35, the GGP region - Gly54, Gly55, Pro56 Thr60, Gly61), located in loops CD, EF and to the pre-F region, allow larger flexibility to the polypeptide chain (glycines especially) and the bigger fluctuations predicted by the model are due to their diminished connectivity as well, being the most exposed to the solvent. This was expected, since the model totally neglects solvent exposure.

The simplified approach used here shows a remarkably better agreement with experiment, for buried regions, where the connectivity of atoms is greater and the solvent plays a minor role.

\section{RESULTS AND DISCUSSION}

In order to identify the relevant motions of the protein the reduced covariance matrix plot (figure 3) of $\mathrm{PtrHb}$ is inspected (PtrHb will be the main target of the following discussion, the same considerations holding for $\mathrm{CtrHb}$ as well), normalized as follows:

$$
c_{i j}=\frac{\left\langle\delta \mathbf{r}_{i} \cdot \delta \mathbf{r}_{j}\right\rangle}{\sqrt{\left\langle\delta \mathbf{r}_{i} \cdot \delta \mathbf{r}_{i}\right\rangle\left\langle\delta \mathbf{r}_{j} \cdot \delta \mathbf{r}_{j}\right\rangle}}
$$

Normalization is generally performed in order to allow a direct comparison between the cross-correlations predicted by the model and the ones obtained in computer simulations, e.g. from molecular dynamics, provided equilibration has been reached [4].

From the reduced normalized covariance matrix one is able to extract non trivial informations on the collective motions of the protein under study: these generally involve the regions of the molecule that show negative correlations.

Indeed it turns out that spatially closed parts of the molecule, i.e. residues in contact, undergo motions with positive correlation, as one would expect for contact-driven motions.

One can identify three main blocks in the covariance plot (fig. [3): the first one contains helices A, B, C, E, the loops between them and the EF loop; the second one includes clearly 


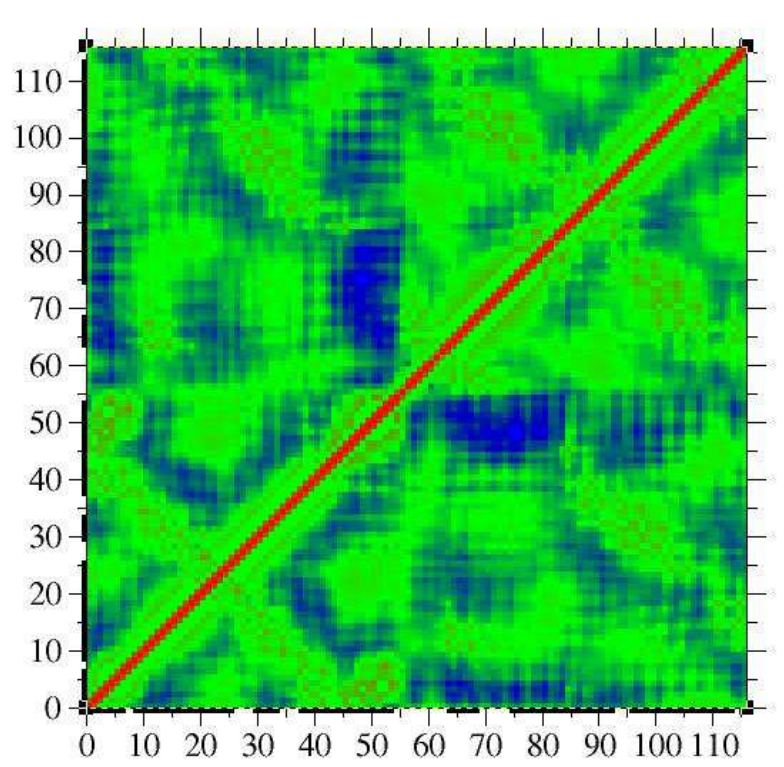

FIG. 3: Normalized covariance matrix: trivial correlations due to contacts have been put to 0 (green); diagonal elements are equal to 1 (red); anti correlation range from 0 to the minimum value found, for Gln41 (E7) and His68 (F8), lower then -0.35 (blue). Helical regions have been highlighted.

the preF-loop, heme bound helix F, as well as the first part of helix G, while the third block hosts the major part of helix $\mathrm{G}$ and the C-terminal side of helix $\mathrm{H}$.

Most residues in the first block, especially the ones belonging to helical regions $\mathrm{A}, \mathrm{B}$ and E (distal side), show a remarkable anti-correlation with residues localized at the beginning of the second block, belonging to the proximal helix F and to helix G; in the third block the last turns of helix $\mathrm{H}$ is bent at the C-terminal to allow closer contacts with heme [21].

This division in domain of motions is similar to the one found in [41] for deoxymyoglobin, provided that one notes the effect of the bending of C-terminal side in helix $\mathrm{H}$, which implies a correlated motion with the proximal side, as suggested by fig. 固 where normalized correlations between His68 (F8) and the rest of the protein are shown. Here the crucial role of small helix $\mathrm{F}$ in the dynamics of the protein is underlined, since it contains the proximal histidine, and the division of the protein in domains of motion as described above is made more evident.

The covariance minima in the plot of figure 4 are particurlarly meaningful, being found 


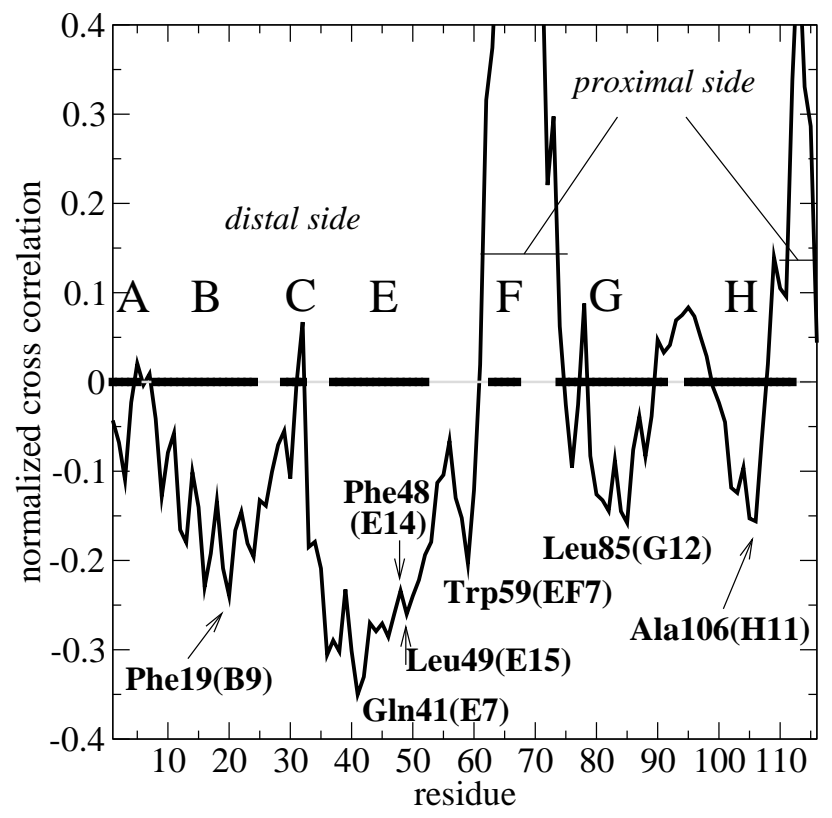

FIG. 4: Normalized cross correlations between the proximal histidine F8 and the rest of the protein $\left(c_{68 j}\right.$, hence the peak raising to 1.0 at $\left.j=68\right)$ : residues displaying significant anti-correlations with His68 are labeled in the plot. Like Phe19 (B9), they are strongly conserved throughout the trHb family [21], being relevant to prevent solvent access to the heme pocket (E14, EF7 [21]), to stabilize heme bound ligand (E7 21] ) and to build the gate between the heme pocket and an apolar cavity running inside the protein matrix (G12, H11 28] ).

between His F8 and other key residues of the protein. Phe19 (B9), which has a bulky side chain, is responsible for the screening of the distal cavity from the acqueous environment outside the molecule, and is strongly conserved among trHbs; in a position occupied by the distal histidine in vertebrate Hbs we found Gln41 (E7), hydrogen bonded to Tyr20 (B10), which contribute to stabilize the heme-bound ligand 21] and form a hydrogen bonding network in the heme pocket, which is believed to be responsible for the different ligand rebinding kinetics displayed by $\mathrm{PtrHb}$ and $\mathrm{CtrHb}$ in comparison with Mbs and $\mathrm{Hbs}$ [48].

His68, taken here as a representative to deduce the motion of the whole proximal side from the covariance and correlation plots (figg. (3. 4), anti-correlates with hydrophobic Phe33 (CD1) as well, another strongly conserved residue among trHbs: together with the previous three residues they line precisely the distal cavity facing the heme group. The anti-correlation of the distal and proximal sides is a clear sign of the concerted motion which may allow the 
heme pocket to expand, thus making easier access to heme for ligands coming from the apolar cavity that links the inner part of the protein to the solvent [21], escaping the steric hindrance of the distal side residues.

Strong anti-correlation with the proximal histidine are displayed by Leu49 (E15), Leu85 (G12) and Ala/Val106 (H11) as well: these residues lie at the bottom of the distal cavity, at the interface between the tunnel running inside the protein matrix and the heme pocket 28 , 49].

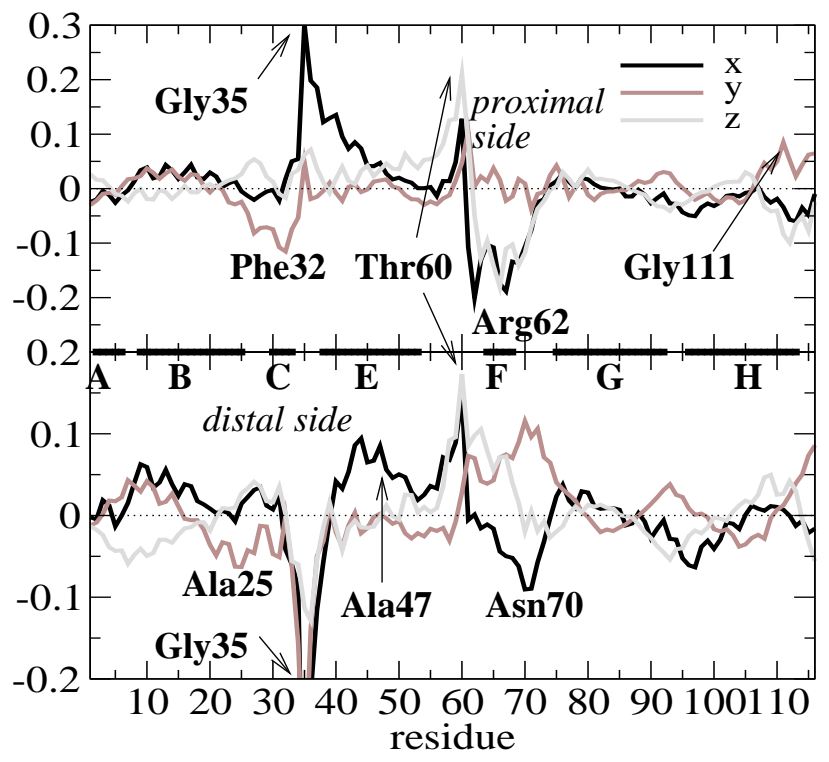

FIG. 5: Components of normalized eigenvectors for the first two slowest modes of motion (1, top; 2, bottom; ratio of corresponding eigenvalues: 1.16), which bring a similar contribute to the dominant opening mechanism of the distal cavity, driven by the anticorrelated motions of the proximal (pre-F loop, helix F, loop FG and last part of helix H) and distal sides (helix C, CD loop and helix E especially). Residues with bulky side chains, strongly conserved in the family of trHbs and belonging to the hydrophic cluster preventing solvent access to the heme pocket 21] are spatially located near the residues with biggest components, highlighted in the plot: Phe33 (CD1), Trp59 (EF7), Phe48 (E14). The latter acts as gating residue in trHbN from Mycobacterium Tuberculosis 49].

The anticorrelated motion of the proximal and distal sides is made more visible by inspection of the components of the eigenvectors corresponding to the first two slowest overdamped modes, plotted in figure 5 
Residues displaying the biggest deviations from their native positions are highlighted: they belong to loops between helices lining the heme pocket (CD and EF loops, pre-F region), and to helices enclosing the distal and proximal sides (helix B and E, helix F and H). These modes contribute substantially to the opening and closing of the distal side, in agreement with previous studies on globins [41, 42].

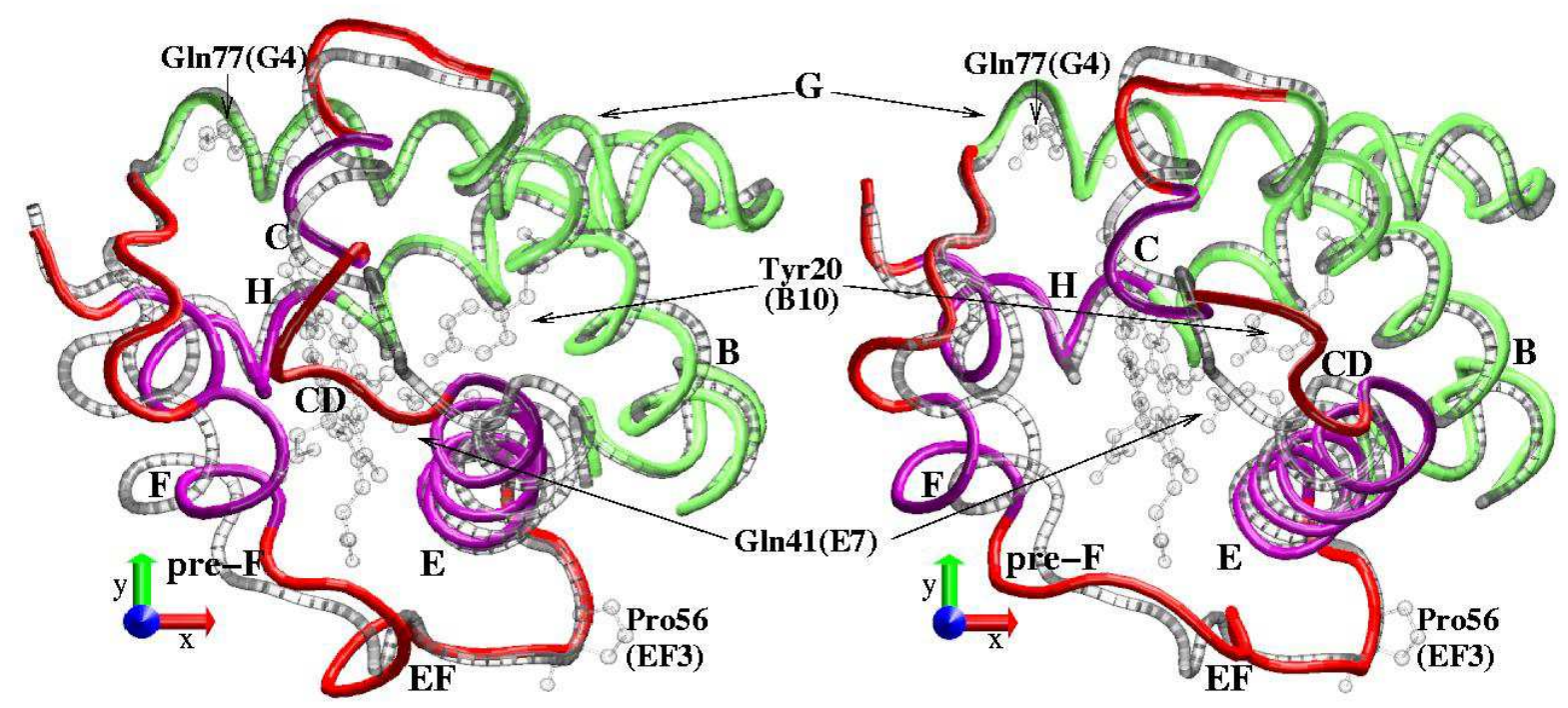

FIG. 6: Open (left) and closed (right) conformations of the distal cavity, obtained by adding or subtracting the rescaled eigenvector of the first slowest mode to the native positions of $\alpha$ carbons (scaling factor: 20). Most mobile regions in the first mode are coloured in red (loops) and purple (helices). Heme group and native structure are drawn in gray, as well as heme bound ligand stabilizing residues Tyr B10 and Gln E7 and hinges of the distal side opening mechanism - Pro EF3 and Gln G4 (figure drawn using VMD [50] and Raster3d [33]).

A detailed view of the conformations visited by the first mode is shown in figure [ where the open and closed structures of the distal cavity are displayed, along with distal residues Tyr B10 and Gln E7.

From the covariance plot (fig. 3i) and the component along the y axis of the second slowest eigenvector of figure 5 (although small, due to the normalization, which enhances most mobile regions like loops) one can notice the anticorrelation of the proximal histidine with the residues identified to line the passage leading to the heme pocket from the tunnel inside 
the protein (mainly Phe19, Leu85 and Ala106, already evidenced in fig. (4) [24, 28, 449, 51]. The anti-correlation between the two groups of residues hints at a possible mechanism for the passage of ligands to the heme pocket, through the enlargement of the gate: the presence of the apolar cavity has been proposed to contribute effectively to the fast rebinding of ligands on heme, together with the hydrogen bonding network in the distal side, as already pointed out [24, 28, 48, 51, 52].

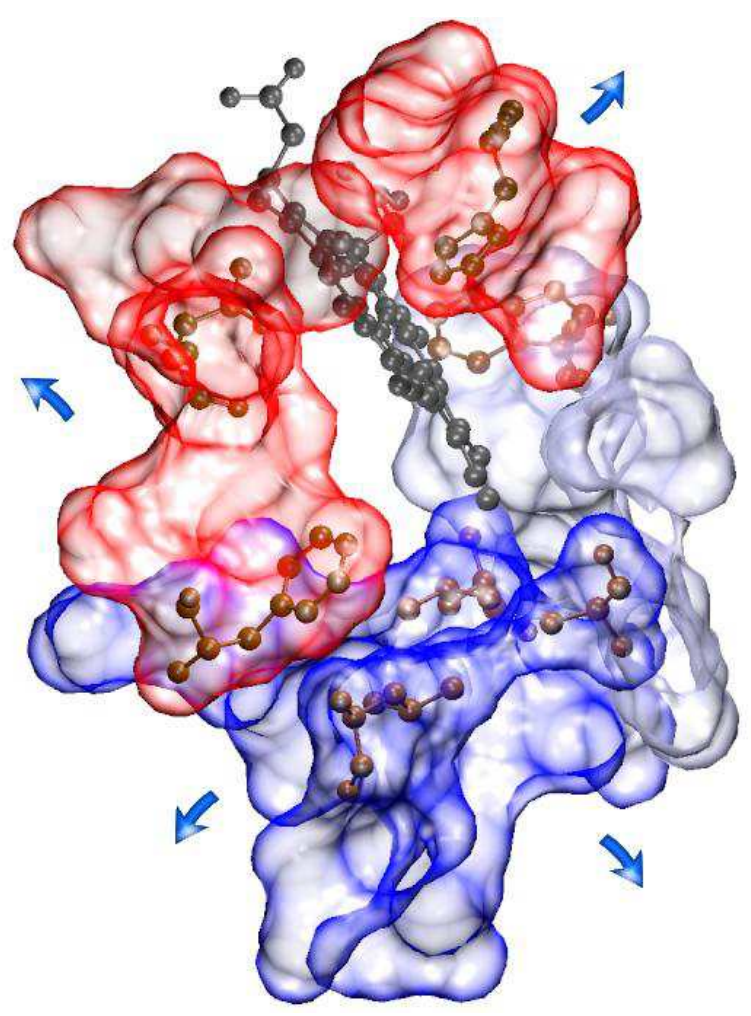

FIG. 7: Schematic representation of near equilibrium motions of groups of residues delimiting the heme pocket, inferred from covariance analysis: solvent accessible surfaces of residues delimiting the apolar cavity (res. $6,12,16,17,49,53,85,89$, lower left), and the distal cavity (proximal side: res. $64,68,71$, upper right; distal side: res. 19, 20, 32, 33, 41, upper left) are shown with a $1.4 \AA$ radius probe. Ball-and-stick representation is used for His68 and the residues labeled in figure 4. The cluster in the lower right (res. 48, 51, 52, 59, 105, 109) defines a narrower cavity [51]. Figure drawn with VMD [50], rendered with Raster3d [33].

The combined motion of the main blocks is compatible with a pumping mechanism: according to the results obtained in this study, the native state conformation of the two 
truncated hemoglobins is such that small displacements of the atoms, due to stochastic interactions with the solvent, produce an anti-correlated motion of the proximal and distal sides, which line the heme pocket, bringing atoms back to equilibrum positions. These movements may facilitate the diffusion of small ligands such as $\mathrm{O}_{2}$ and $\mathrm{CO}$ to heme through the protein tunnel, exploiting its volume variations [49].

\section{A. Elasticity and time scale}

An estimate of the elastic constant $\gamma$ of the model can be computed fitting the experimental temperature factors of the X-ray structures with the theoretical ones, obtained from the mean square displacements of $C^{\alpha}$ s, according to equation (10). Following the method used in 12] to fit the data (i.e. by matching the areas of the surface enclosed by the two data sets) and averaging the values found for the two proteins yields $\gamma=0.20 \mathrm{Nm}^{-1}$, with a tolerance of $0.05 \mathrm{Nm}^{-1}$ between averaged values (the introduction of heme in the network of interactions leads to a decreas of the value of the elastic constant, since it enhances the local connectivity of the buried residues in the heme pocket). The order of magnitude obtained for $\gamma$ agrees with estimated values for the elastic constant of single parameter models [10, 15, 19, 20].

The importance of friction due to the solvent in determining the rates of functional motions of proteins, as it slows down the relaxation times of large-scale displacements predicted by normal mode analysis, has recently been underlined [53] : in the framework of the Langevin dynamics introduced with equation (8), we estimate order of magnitude for the reference decay time $\tau$ of the first two modes of motion previously described, through an effective value for the friction coeffient $f$, chosen to be the same for all residues for simplicity. A lower limit for $f$ is the value computed from an all-atom simulation in [54], whereas here whole residues are considered (although the effective radii associated with such an estimate are bigger than the Van der Waals radii of the atoms in the simulation, hinting at a collective character of the simulated displacements [54], the motions predicted by the slowest modes involve many more residues in distant parts of the protein and a larger value for the friction may be expected). As an upper limit, the friction relative to the whole proteins (both $\mathrm{PtrHb}$ and $\mathrm{CtrHb}$ roughly fit a cubic box of side $3.5 \mathrm{~nm}$ ) moving in water at physiological conditions is calculated from Stoke's law (see [55], chapter 3). We obtained $f \sim 4 \div 70 \mathrm{pN} \mathrm{m}^{-1} \mathrm{~s}$ (similar ranges for the values of friction coefficients have been extracted from molecular 
dynamics simulations [56]).

The corresponding reference relaxation time $\tau$ in the Langevin dynamics of equations 8 and 9 lies within the range $0.02 \div 0.35 \mathrm{~ns}$, while the relaxation time associated with eigenmode $i$ will be $\tau_{i}=\frac{\tau}{\lambda_{i}}$ (where $\lambda_{i}$ is the eigenvalue relative to that eigenmode): the two slowest eigenmodes display relaxation times for the related motions approximately within the range $0.2 \div 3.5 \mathrm{~ns}$.

This range of time scale is compatible with CO rebinding kinetics of Mbs and Hbs, while $\mathrm{PtrHb}$ and $\mathrm{CtrHb}$ behave quite differently [48]: the explanation proposed for the different behaviour relies on the hydrogen-bonding network formed in the distal cavity of these trHbs, which is absent in invertebrate globins and is beyond the possibility of the simple model used here, which underlines instead the common characteristics of globins and trHbs.

\section{CONCLUSIONS}

It has been shown how a simple coarse-grained approach can bring insights into the functional motions of two small proteins of the truncated hemoglobins family, PtrHb and $\mathrm{CtrHb}$, near equilibrium vibrational properties of the structures modeled as a gaussian network of interacting $\alpha$ carbons and $\beta$ centroids.

The key point in the analysis performed here is the information extracted from the covariance matrix in its reduced form and from the two slowest modes of fluctuation: negative correlations between residues set far apart in the tridimensional structure are particularly useful, being non trivial and hinting at the collective character of the motions.

This information has been used in the present work to confirm within such a simplified approach the mechanism which is believed to facilitate small ligands diffusion to the heme pocket and the iron atom. The cavity delimited by several key hydrophobic residues, providing a path from the surface of the protein to the heme pocket [21, 24, 28, 52], is able to enlarge its volume allowing the passage of small molecules to the distal side [48, 49], as it is inferred from the anti-correlations between the displacements of the opposite sides of the heme pocket.

Excitations, due to interactions between the molecule and the solvent, produce deviations from equilibrium followed by a decay towards the native state. The collective behaviour of the return back to equilibrium, produced by a superposition of overdamped motions, allow 
the volume of the inner cavities to vary accordingly.

Through a fit of the mean square displacements of $\alpha$ carbons from their minimum energy configuration with the experimental temperature factors for the two structures under study, a rough estimate of the order of magnitude of time scale for functionally relevant motions has been given, in reasonable agreement with known properties of globular proteins.

This suggests the validity of the simple gaussian approach as a means to get a fast picture of the near-native functional motions of globular proteins, yet in agreement with the results obtained using more accurate and computationally demanding tools.

The description given by the simple model used here does not provide atomic details, keeping the analysis at a coarse-grained level. Still the use of the effective $\beta$ centroid for each residue, along with the $C^{\alpha}$, helps in characterizing with more adherence to reality the displacements of residues side-chains, thus getting a closer agreement with more detailed approaches.

\section{ACKNOWLEDGMENTS}

I wish to thank Amos Maritan and Gianluca Lattanzi for the invaluable suggestions and the critical reading of the manuscript. The collaboration with Cristian Micheletti in the initial stage of this work is gratefully acknowledged.

[1] T. Noguti and N. Gō, Nature 296, 776 (1982).

[2] N. Gō, T. Noguti, and T. Nishikawa, Proc. Natl. Acad. of Sci. USA 80, 3696 (1983).

[3] B. Brooks and M. Karplus, Proc. Natl. Acad. of Sci. USA 80, 6571 (1983).

[4] M. Levitt, C. Sander, and P. S. Stern, Int. J. Quant. Chem.: Quant. Biol. Symp 10, 181 (1983).

[5] M. Levitt, C. Sander, and P. S. Stern, J. Mol. Biol. 181, 423 (1985).

[6] S. Hayward and N. Gō, Annu. Rev. of Phys. Chem. 46, 223 (1995).

[7] S. Hayward, A. Kitao, and N. Gō, Proteins Sci. 3, 936 (1994).

[8] A. Amadei, A. B. M. Linssen, and H. J. C. Berendsen, Proteins: Structure, Function, and Genetics 17, 412 (1993). 
[9] T. Horiuchi and N. Gō, Proteins: Structure, Function, and Genetics 10, 106 (1991).

[10] M. Tirion, Phys. Rev. Lett. 77, 1905 (1996).

[11] T. Haliloglu, I. Bahar, and B. Erman, Phys. Rev. Lett. 79, 3090 (1997).

[12] I. Bahar, A. R. Atilgan, and B. Erman, Fold. Des. 2, 173 (1997).

[13] I. Bahar, A. R. Atilgan, M. C. Demirel, and B. Erman, Phys. Rev. Lett. 80, 2733 (1998).

[14] P. Doruker, A. R. Atilgan, and I. Bahar, Proteins: Structure, Function, and Genetics 40, 512 (2000).

[15] A. R. Atilgan, S. R. Durell, R. L. Jernigan, M. C. Demirel, O. Keskin, and I. Bahar, Biophys. J. 80, 505 (2001).

[16] P. Doruker, R. L. Jernigan, and I. Bahar, J. Comput. Chem. 23, 119 (2001).

[17] F. Tama and Y.-H. Sanejouand, Protein Eng. 14, 1 (2001).

[18] F. Tama, J. F. M. Valle, and C. L. Brooks, Proc. Natl. Acad. Sci. USA 100, 9319 (2003).

[19] C. Micheletti, P. Carloni, and A. Maritan, Proteins: Structure, Function, and Bioinformatics $\mathbf{5 5}, 635(2004)$.

[20] M. Neri, M. Cascella, and C. Micheletti, Influence of conformational fluctuations on enzymatic activity: modelling the functional motion of beta-secretase (in press), j. Phys. Cond. Mat.

[21] A. Pesce, M. Couture, S. Dewilde, M. Guertin, K. Yamauchi, P. Ascenzi, L. Moens, and M. Bolognesi, The EMBO Journal 19, 2424 (2000).

[22] M. Couture, S. Yeh, B. A. Wittenberg, J. B. Wittenberg, Y. Ouellet, D. L. Rousseau, and M. Guertin, Proc. Natl. Acad. Sci. USA 96, 11223 (1999).

[23] S. Yeh, M. Couture, Y. Ouellet, M. Guertin, and D. L. Rousseau, Proc. Natl. Acad. Sci. USA 96, 11223 (1999).

[24] J. B. Wittemberg, M. Bolognesi, B. A. Wittenberg, and M. Guertin, J. Biol. Chem. 277, 871 (2001).

[25] M. Potts, S. V. Angeloni, R. E. Ebel, and D. Bassam, Science 256, 1690 (1992).

[26] M. V. Thorsteinsson, D. R. Bevan, M. Potts, Y. Dou, R. F. Eich, M. S. Hargrove, Q. H. Gibson, and J. S. Olson, Biochemistry 38, 2117 (1992).

[27] M. Couture, H. Chamberlan, B. St-Pierre, J. Lafontaine, and M. Guertin, Mol. Gen. Genet. 243, 185 (1994).

[28] M. Milani, A. Pesce, Y. Ouellet, P. Ascenzi, M. Guertin, and M. Bolognesi, The EMBO Journal 20, 3902 (2001). 
[29] M. Brunori and Q. H. Gibson, EMBO Rep. 2, 674 (2001).

[30] F. Schotte, M. Lim, T. A. Jackson, A. V. Smirnov, J. Soman, J. S. Olson, G. N. P. Jr., M. Wulff, and P. A. Anfinrud, Science 300, 1944 (2001).

[31] M. Teeter, Protein Science 13, 313 (2004).

[32] P. J. Kraulis, J. Appl. Cryst. 24, 946 (1991).

[33] E. Merritt and D. Bacon, Methods in Enzymology 277, 505 (1997).

[34] M. F. Perutz, Annu. Rev. Biochem. 48, 327 (1979).

[35] L. Holm and C. Sander, FEBS Lett. 315, 301 (1993).

[36] M. W. King, Biochemistry of amino acids, http://web.indstate.edu/thcme/mwking/aminoacids.html, IU School of Medicine (2003).

[37] B. Park and M. Levitt, J. Mol. Biol. 258, 367 (1996).

[38] M. Doi and S. F. Edwards, The theory of polymer dynamics (Clarendon Press, Oxford, UK, 1986).

[39] W. H. Press, S. A. Teukolsky, W. T. Vetterling, and B. P. Flannery, Numerical Recipes: The Art of Scientific Computing (Cambridge University Press, Cambridge, UK, 1999).

[40] L. Stryer, Biochemistry (W. H. Freeman and Company, New York, NY, 1995).

[41] Y. Seno and N. Gō, J. Mol. Biol. 216, 95 (1990).

[42] Y. Seno and N. Gō, J. Mol. Biol. 216, 111 (1990).

[43] C. Xu, D. Tobi, and I. Bahar, J. Mol. Biol. 333, 153 (2003).

[44] C. Micheletti, G. Lattanzi, and A. Maritan, J. Mol. Biol. 321, 909 (2002).

[45] B. Halle, Proc. Natl. Acad. Sci. USA 99, 1274 (2002).

[46] F. Fraternali and L. Cavallo, Nucl. Acids Res. 30, 2950 (2002).

[47] B. Hess, Phys. Rev. E 65, 31910 (2002).

[48] U. Samuni, D. Dantsker, A. Ray, J. B. Wittenberg, B. A. Wittenberg, S. Dewilde, L. Moens, Y. Ouellet, M. Guertin, and J. M. Friedman, J. Biol. Chem. 278, 27241 (2003).

[49] A. Crespo, M. A. Martí, S. G. Kalko, A. Morreale, M. Orozco, J. L. Gelpi, F. J. Luque, and D. A. Estrin, J. Am. Chem. Soc. 127, 4433 (2005).

[50] W. Humphrey, A. Dalke, and K. Schulten, J. Molec. Graphics 14, 33 (1996).

[51] M. Milani, A. Pesce, Y. Ouellet, S. Dewilde, J. Friedman, P. Ascenzi, M. Guertin, and M. Bolognesi, J. Biol. Chem. 279, 21520 (2004).

[52] M. Milani, P. Y. Savard, H. Ouellet, P. Ascenzi, M. Guertin, and M. Bolognesi, Proc. Natl. 
Acad. Sci. USA 100, 5766 (2003).

[53] J. Ma, Structure 13, 373 (2005).

[54] S. Swaminathan, T. Ichiye, W. van Gunsteren, and M. Karplus, Biochemistry 21, 5230 (1982).

[55] J. Howard, Mechanics of motor proteins and the cytoskeleton (Sinauer Associates, Sunderland, MA, 2001).

[56] K. Hinsen, A. J. Petrescu, and S. Dellerue, Chem. Phys. 261, 25 (2000). 